\title{
Congenital unilateral hypoplasia of depressor anguli oris
}

INSERM

\section{Source}

INSERM. (1999). Orphanet: an online rare disease and orphan drug data base. Congenital unilateral hypoplasia of depressor anguli oris. ORPHA:1166

Congenital unilateral hypoplasia of depressor anguli oris is a congenital anomaly, characterized by the unilateral hypoplasia/agenesis of the depressor anguli oris muscle, resulting in an asymmetric crying facies in neonatal period/ infancy (drooping of one corner of the mouth during crying) while eye closure, nasolabial fold and forehead wrinkling are symmetric. While it can be isolated, this anomaly is also seen in 22 q11.2 deletion syndrome (see this term) and can be accompanied by other major congenital anomalies of the cardiovascular system, as well as less frequently the musculoskeletal, cervicofacial, respiratory, genitourinary, and, rarely, endocrine systems. When isolated, the condition is cosmetically insignificant as the infant gets older (as the muscle does not contribute significantly to facial expression in childhood/ adulthood). 